\title{
Investigation of Excitonic Effects in Polar InGaN/GaN Quantum Heterostructures for Enhanced Quantum Electroabsorption in Blue
}

\author{
Emre Sari $^{1,3}$, Sedat Nizamoglü ${ }^{2,3}$, Tuncay Ozel ${ }^{2,3}$, Asli Koc ${ }^{3}$, In-Hwan Lee ${ }^{4}$, Jong Hyeob Baek ${ }^{5}$, and \\ Hilmi Volkan Demir ${ }^{1,2,3}$ \\ ${ }^{1}$ Department of Electrical and Electronics Engineering, ${ }^{2}$ Department of Physics, ${ }^{3}$ Nanotechnology Research Center, \\ Bilkent University, Bilkent, Ankara 06800 Turkey \\ ${ }^{4}$ School of Advanced Materials Engineering, Research Center of Industrial Technology, Chonbuk National University, Chonju 561-756, Korea \\ ${ }^{5}$ Korea Photonics Technology Institute, Gwangju 500-460, Korea \\ Tel:[+90](312) 290 1021, e-mail: volkan@bilkent.edu.tr
}

Excitonic resonance plays a critical role for the enhancement of quantum confined Stark effect in quantum heterostructures [1]. Among the basic design parameters that affect the strength of excitonic effects are the ratio of the well width to the barrier width and the difference between the alloy concentration in the well and the barrier materials. For the polar III-Nitride quantum heterostructures, these two parameters additionally change significantly the strength of the polarization induced electrostatic fields inside the wells and barriers, resulting in the formation of a zig-zag potential profile. Depending on the heterostructure design, these fields may further ionize the excitons and thus diminish the excitonic effects in the associated quantum processes. In this work, we demonstrate strong quantum electroabsorption behavior in polar $\mathrm{InGaN} / \mathrm{GaN}$ quantum heterostructures by carefully designing and controlling the quantum structure parameters and thus obtaining strong excitonic effects for the end goal of making a high-performance quantum electroabsorption modulator in blue.

In III-N multiple quantum well structures, the material polarization fields and built-in electrostatic fields inside the well and barriers are given as in Ref. [2]:

$$
\begin{gathered}
\mathbf{E}_{\text {tot }}^{\text {well }}=\mathbf{E}_{s p}^{\text {well }}+\mathbf{E}_{p z}^{\text {well }}=\frac{\left(\mathbf{P}_{s p}^{\text {barrier }}+\mathbf{P}_{p z}^{\text {barrier }}\right)-\left(\mathbf{P}_{s p}^{\text {well }}+\mathbf{P}_{p z}^{\text {well }}\right)}{\epsilon_{\text {well }}+\frac{d_{\text {well }}}{d_{\text {barrier }}} \epsilon_{\text {barrier }}} \\
\mathbf{E}_{\text {tot }}^{\text {barrier }}=\mathbf{E}_{s p}^{\text {barrier }}+\mathbf{E}_{p z}^{\text {barrier }}=\frac{\left(\mathbf{P}_{s p}^{\text {well }}+\mathbf{P}_{p z}^{\text {well }}\right)-\left(\mathbf{P}_{s p}^{\text {barrier }}+\mathbf{P}_{p z}^{\text {barrier }}\right)}{\epsilon_{\text {barrier }}+\frac{d_{\text {barrier }}}{d_{\text {well }}} \epsilon_{\text {well }}}
\end{gathered}
$$

where $\mathrm{E}, \mathrm{P}, \epsilon$ and $\mathrm{d}$ represent the electric field, polarization, dielectric constant and width, respectively. Here $\mathrm{P}_{\mathrm{sp}}$ corresponds to the spontaneous polarization and $\mathrm{P}_{\mathrm{pz}}$ to the piezoelectric polarization. As given in Eq. (1) [and (2)], the field inside the well (barrier) decreases (increases) with the increasing well-to-barrier width ratio. Also increasing alloy concentration difference directly increases the strength of the built-in fields. The fields inside the well and the barrier may cause photogenerated excitons to be ionized either directly (if the field inside the well is sufficiently large) or via tunneling (if the net field inside the barrier is sufficiently large). The epitaxial structure can, however, be designed such that an externally applied field (e.g., reverse bias) can compensate for the field inside the well, e.g., by growing Ga-face $\mathrm{GaN}$ on c-plane sapphire and p-type layer on the top of the p-i-n architecture.

In principle, the strength of excitonic effects can be adjusted by controlling the Coulomb interaction and overlap of the electron and hole wavefunctions engineering the band diagram. By designing an epitaxial structure that allows for the compensation of the field inside the well by the electric field externally applied in the opposite direction of the polarization in the wells, photogenerated electron and hole pair gets relatively closer to each other despite the polarization field and provide an enhancement in the strength of excitonic effects. However, this also eases the tunneling of the carriers since the external field is then in the same direction with the polarization field inside the barrier. Therefore, the barrier becomes effectively thinner in potential and a transition from the quantum structure behavior to the bulk behavior is observed in the optical absorption spectra at increasing external fields (Figure 1). Although it is possible to decrease the field inside the well structure by increasing the well-to-barrier width ratio, this introduces difficulties in the growth process, and again facilitates tunneling through the effectively thinner barriers. Hence, to realize strong quantum electroabsorption, we design the quantum structures carefully with an optimal alloy concentration discontinuity and an optimal well-to-barrier width ratio.

To realize high-performance electroabsorption modulators, we grew, fabricated, and investigated $2.5 \mathrm{~nm} / 7.5 \mathrm{~nm}$ and $4 \mathrm{~nm} / 4 \mathrm{~nm} \operatorname{In}_{\mathrm{x}} \mathrm{Ga}_{1-\mathrm{x}} \mathrm{N} / \mathrm{GaN}$ structures $(\mathrm{x} \sim 0.12)$ developed to operate in blue $(\lambda=420-440 \mathrm{~nm})$ both with a PL peak of $440 \mathrm{~nm}$ and $2.5 \mathrm{~nm} / 8 \mathrm{~nm} \operatorname{In}_{\mathrm{x}} \mathrm{Ga}_{1-\mathrm{x}} \mathrm{N} / \mathrm{GaN}$ structures $(\mathrm{x} \sim 0.25)$ to operate in cyan $(\lambda=470-490 \mathrm{~nm})$ with a PL peak of $530 \mathrm{~nm}$. All the wafers were grown on Ga-face c-plane sapphire substrates in MOCVD (metallo organic chemical vapour deposition). The resulting $\mathrm{p}$-i-n diodes were fabricated in surface-normal mesa architecture with a p-layer grown on top of the structure. The main fabrication steps consisted of reactive ion etching, metallization, and annealing. $\mathrm{Ni} / \mathrm{Au}$ and $\mathrm{Ti} / \mathrm{Al}$ were used to form the ohmic contacts to $\mathrm{p}$ - and $\mathrm{n}$-layers, respectively. The structures 
were tested in an electroabsorption test setup that includes a continuous Xe lamp, chopper, monochrometer, lock-in amplifier and a power supply. The incoming optical power was tracked using an optical power meter. Fig. 1 presents the electroabsoprtion behavior of these polar InGaN/GaN quantum structures. The first InGaN/GaN $2.5 \mathrm{~nm} / 7.5 \mathrm{~nm}$ quantum structure operating in the blue range exhibits a Franz-Keldysh like behavior with an inflection point around $430 \mathrm{~nm}$ as presented in Fig. 1a and Fig. 1b. These electroabsorption spectra show a blueshift as a result of the "reversed" Franz-Keldysh effect due to the excitons that are already ionized by the built-in electrostatic fields inside the wells when no external field is applied. Here the absorption coefficient change is minimal due to the diminished excitonic effects. As depicted in Fig. 1c and Fig. 1d, the second InGaN/GaN $2.5 \mathrm{~nm} / 8 \mathrm{~nm}$ quantum structure operating at $\sim 490 \mathrm{~nm}$ also exhibits a Franz-Keldysh like behavior with a clear inflection point around $470 \mathrm{~nm}$. The structure shows a blueshift owing to the reversed Franz-Keldysh effect because the excitons are ionized by the builtin electrostatic fields inside the wells due to the low well-to-barrier width ratio and high alloy concentration difference. Here the absorption coefficient change is even smaller due to further diminishing excitonic effects. On the other hand, in Fig. 1e and Fig. 1f, the InGaN/GaN $4 \mathrm{~nm} / 4 \mathrm{~nm}$ structure exhibits a clear excitonic shoulder around $420 \mathrm{~nm}$ that gives rise to the reversed quantum confined Stark effect with a blueshift by the external field compensating for the built-in electrostatic field inside the well structures. This structure also features the highest absorption coefficient change ( $6000 \mathrm{~cm}^{-1}$ corresponding to $50 \mathrm{~cm}^{-1}$ per $1 \mathrm{~V} / \mu \mathrm{m}$ E-field swing) that is comparable to other III-V quantum structures with square potential profiles. This structure exhibits the transition from quantum to bulk behavior in the optical absorption spectra with the increasing external bias only beyond 6V [3].

In conclusion we investigated excitonic effects in polar InGaN/GaN quantum structures to achieve strong quantum electroabsorption effect in blue and demonstrated the highest absorption coefficient change (about $6000 \mathrm{~cm}^{-1}$ ) in blue due to the reversed quantum confined Stark effect with the realization of $4 \mathrm{~nm} / 4 \mathrm{~nm} \mathrm{InGaN} / \mathrm{GaN}$ structure.
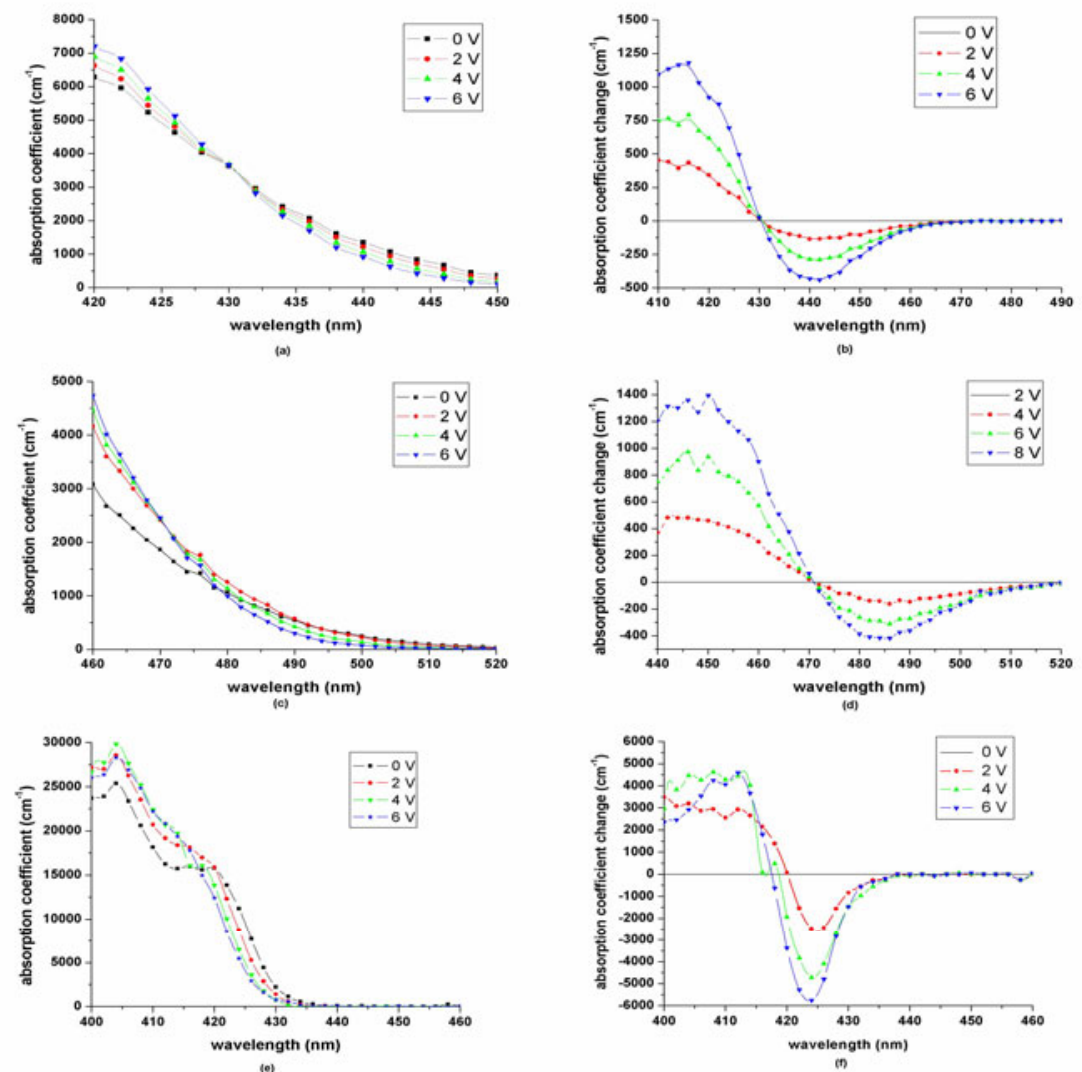

Figure 1 Optical absorption spectra and absorption coefficient change for InGaN/GaN structures: $a, b) 2.5 \mathrm{~nm} / 7.5 \mathrm{~nm}$ operating in blue; $c$, $d$ ) 2.5 $\mathrm{nm} / 8 \mathrm{~nm}$ operating in cyan and $e, f) 4 \mathrm{~nm} / 4 \mathrm{~nm}$ operating in blue.

Acknowledgements: This work is supported by EU MOON, EU NOE PHOREMOST and TUBITAK 104E114, 106E020, 105E065, and 105E066. H.V.D., E.S., and S.N. also acknowledge additional support from Turkish Academy of Sciences (TUBA GEBIP) and TUBITAK.

References

[1] D.A.B. Miller, et al., Phys. Rev. B, 32, 1043 (1985).

[2] V. Fiorentini, et al., Phys. Rev. B, 60, 8849 (1999).

[3] E. Sari, et al., Appl. Phys. Lett., 90, 011101 (2007). 